\title{
Effect of viscous dissipation in two-dimensional Stokes flow between rotating cylinders - preliminary numerical investigation
}

\author{
Tomasz Janusz Teleszewski ${ }^{1, *}$ \\ ${ }^{1}$ Department of HVAC Engineering, Faculty of Civil and Environmental Engineering, Bialystok University of Technology, Wiejska 45A, \\ 15-351 Bialystok, Poland
}

\begin{abstract}
This paper introduces the modelling of two-dimensional laminar flow of Newtonian fluid in a system of concentric or eccentric rotating cylinders with regards to viscous dissipation. Viscous dissipation is a main part, where the viscosity is large for example in oils. The dependence of the Nusselt number on the ratio of the radius of the inner cylinder to the radius of the external cylinder for the selected distance of the cylinder axes was investigated. In order to determine the velocity fields and the temperature distribution, the boundary element method was used. The results of the calculations were presented in the form of diagrams.
\end{abstract}

\section{Introduction}

The problem of mathematical modelling of viscous fluid movement in eccentric rotating cylinder systems (Fig. 1) is interesting in a scientific aspect as a peculiar form of Taylor-Couette flows [1], and extremely important from an engineering point of view in lubrication theory [2] and sliding designs of hydrodynamic radial bearings [2], liquid mixers with different physical properties [3], rotational viscometers [4].

Although the flow of viscous fluid in the space between cylinders (two, or more generally many) is a three-dimensional flow, assuming a considerable length of cylinders, a sufficient approximation is the adoption of the two-dimensional flow model described in the Stokes equations and continuity for a viscous, incompressible fluid.

The viscosity dissipation in the flows between the rotating cylinders is usually neglected. Flows between rotating cylinders are solved by analytical $[1,5,6]$ or numerical [7-11] methods. Considerations of viscous dissipation for flows with a small Reynolds number usually relate to flows in microchannels [12-14]. Examples of the application of the boundary element method for solving the viscous dissipation effect for laminar flow in straight ducts were presented in $[15,16]$. Examples of viscous dissipation solutions in flows between rotating cylinders can be found in the literature [17-19].

The aim of this study is to preliminary investigate the effect of viscous dissipation in Stokes flows of Newtonian fluid between rotating cylinders. The physical model of the discussed problem is presented in Figure 1. The main geometric parameter is the ratio of the radius of the inner cylinder to the radius of the external cylinder $r_{1} / r_{2}$ and the distance between the centres of the cylinders $\varepsilon$.

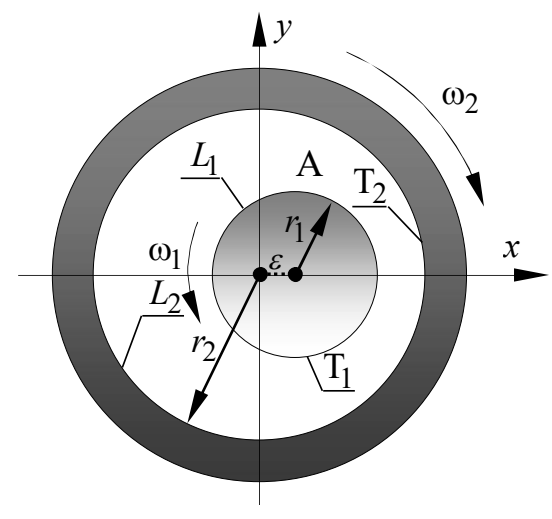

Fig. 1. Flow between two eccentric rotating cylinders in twodimensional problem.

\section{Mathematical formulation}

The presented problem of the effect of viscous dissipation in two-dimensional Stokes flow between rotating cylinders is described by the momentum (1) and the conservation of energy equation (2) with consideration of the viscous dissipation term $\Phi(x, y)$ (3):

$$
\begin{aligned}
& \frac{\partial^{2} u_{x}}{\partial x^{2}}+\frac{\partial^{2} u_{x}}{\partial y^{2}}=\frac{1}{\mu} \frac{\partial p}{\partial x}, \\
& \frac{\partial^{2} u_{y}}{\partial x^{2}}+\frac{\partial^{2} u_{y}}{\partial y^{2}}=\frac{1}{\mu} \frac{\partial p}{\partial y}, \\
& k\left(\frac{\partial^{2} T}{\partial x^{2}}+\frac{\partial^{2} T}{\partial y^{2}}\right)=\Phi(x, y)
\end{aligned}
$$

\footnotetext{
*Corresponding author: t.teleszewski@pb.edu.pl
} 


$$
\Phi(x, y)=-\mu\left[2\left(\frac{\partial u_{x}}{\partial x}\right)^{2}+2\left(\frac{\partial u_{y}}{\partial y}\right)^{2}+\left(\frac{\partial u_{y}}{\partial x}+\frac{\partial u_{x}}{\partial y}\right)^{2}\right]
$$

where $u_{z}$ and $u_{y}$ are a velocity component, $p$ is the pressure, $\mu$ is the dynamic viscosity, $T$ is the temperature and $k$ is the thermal conductivity.

The differential equation 2 includes the temperature condition (Dirichlet condition) and the heat flux density condition (Neumann condition). In this study, energy equation (2) is subjected to the following boundary conditions for the cylinder inner wall and outer wall respectively:

$$
T_{1}=T_{2}=\text { const., at } L_{1} \text { and } L_{2}
$$

It should be noted here that the determined issue, the main role is played by viscous dissipation and the walls of the rotating cylinders are maintain at a constant temperature.

\section{Results and analysis}

In order to perform the simulation, the author's calculative program was written in Fortran language, in which the calculations are carried out in two stages. First, the energy dissipation function from equation (1) is determined, which is the condition for equation (2). In the second part of the program, the equation (2) is solved to determine the temperature field. The calculations were made using the boundary element method (BEM) for 1000 linear elements on the boundary $L=L_{1}+L_{2}$. and 80400 triangular cells in area A. In order to verify the method, the values of temperature fields in area $A$ were compared with the known analytical solution of the temperature field including energy dissipation for the Stokes flow between two concentrically rotating cylinders [1]. For the above assumed number of elements on the $L$ boundary and area $A$, the maximum relative differences in temperature did not exceed $0.001 \%$.

The Nusselt number is equal to

$$
\mathrm{Nu}_{v d}=\frac{q_{t o t} r_{1}}{k\left(T_{w}-T_{m}\right)}
$$

where the mean temperature is defined as

$$
T_{m}=\frac{1}{A} \iint_{A} T(x, y) d A
$$

where $T_{w}$ is the temperature at the boundary $L$ equal to $T_{w}=T_{1}=T_{2}$.

Figure 2 shows of the graphical results of calculations of Nusselt numbers as a function of the geometrical parameter $r_{1} / r_{2}$ with $\varepsilon=0.1$. As the ratio between the radius of the inner cylinder and the radius of the outer cylinder increases, the number of Nusselt increases.
The dimensionless velocity, temperature distribution and dimensionless dissipation function in area $\mathrm{A}$ is obtained from the following formulas, respectively

$$
\begin{gathered}
\vartheta=\frac{u}{u_{m}} \\
\theta=\frac{T}{T_{m}} \\
\varphi=\frac{\Phi}{\Phi_{m}}
\end{gathered}
$$

where, the mean velocity and mean dissipation function is defined as

$$
\begin{aligned}
& u_{m}=\frac{1}{A} \iint_{A} u_{z}(x, y) d A \\
& \Phi_{m}=\frac{1}{A} \iint_{A} \Phi(x, y) d A
\end{aligned}
$$

Figures 3a-c respectively show the velocity field with streamlines, dimensionless temperature with heatlines and viscous dissipation function $\Phi(x, y)$ for $\varepsilon=0.1$ and $r_{1} / r_{2}=0.1$.

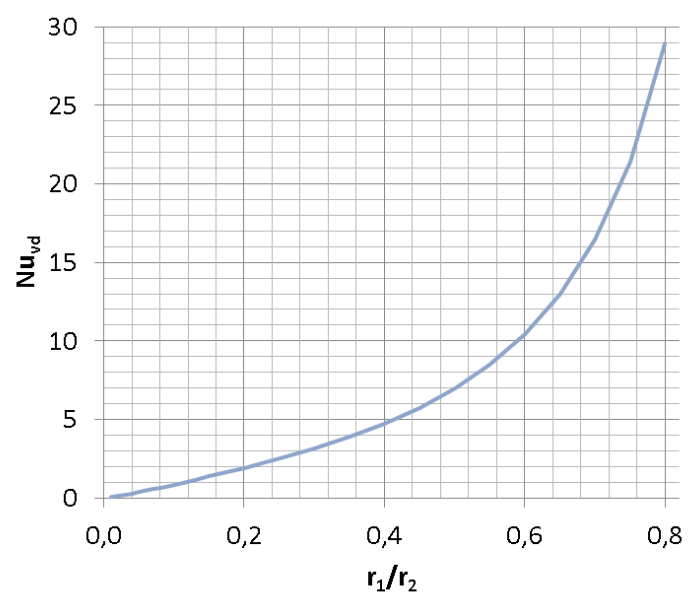

Fig. 2. The influence of $\mathrm{Nu}_{\mathrm{vd}}$, on the $r_{1} / r_{2}$ for $\varepsilon=0.1$.

The results of calculations of velocity fields and streamlines (Fig. 3a) are consistent with the results of calculations from work [6]. A characteristic feature of a given geometry $\left(r_{1} / r_{2}=0.885\right)$ is the creation of a single eddy. The trends of the temperature distribution (Fig. 3b) are consistent with the results of calculations in [17], where the highest temperature increase is located at the narrowest point between the rotating cylinders. In the case of the viscous dissipation function (Fig. 3c) for the given geometry, the maximum value $\Phi(x, y)$ is also at the narrowest point between the rotating cylinders. 
a)

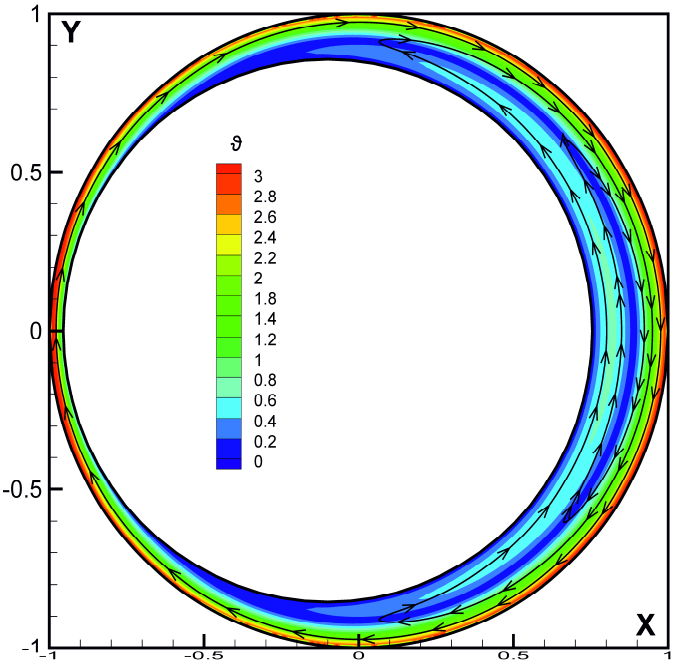

b)

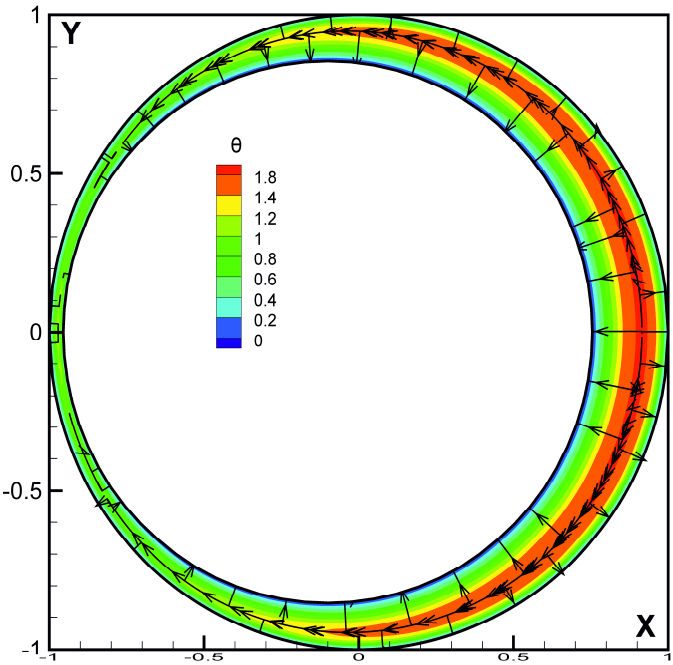

c)

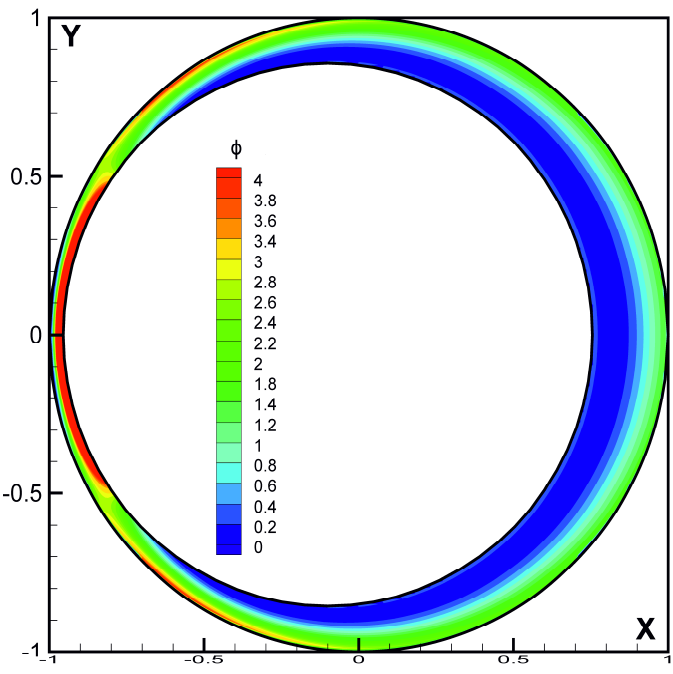

Fig. 3. Graphical results visualization of calculations using the boundary element method for $\omega_{1}=0, \omega_{2} \neq 0$ with $\varepsilon=0.1$ and $r_{1} / r_{2}=0.885$ : a) streamlines and dimensionless velocity, b) heatlines and dimensionless temperature, c) dimensionless dissipation function.

\section{Conclusions}

The above study shows the usefulness of the boundary element method for solving the problems described in Stokes equations, in the analysed case for the determination of a two-dimensional viscous fluid flow including viscous dissipation in a system of concentric or eccentric rotating cylinders at different angular velocities, reduced to the analysis of the flow of a viscous fluid in a plane perpendicular to the cylinder axis. The paper presents the results of calculations using the boundary element method for example with a given geometry. The dependence of the Nusselt number increases with the increase of the radius of the inner cylinder relative to the radius of the outer cylinder.

\section{Acknowledgements}

This work has been carried out in the Department of HVAC Engineering, Faculty of Civil and Environmental Engineering, Bialystok University of Technology, Bialystok, Poland. This scientific project was financed within the framework of science research S/WBiIS funds at Bialystok University of Technology.

\section{References}

1. F.M. White, Viscous Fluid Flow (McGraw-Hill, 2005)

2. M.M. Khonsari, E.R. Booser, Applied Tribology: Bearing Design and Lubrication (John Wiley \& Sons Ltd., 2008)

3. D.G. Thomas, AIChE. J. 10(4), 517 (2009)

4. L. Bin, W. Yu, Procedia Engineering 29, 3513

5. G.I. Taylor, Phil. Trans. Royal Society 223, 289 (1923)

6. B.Y. Ballal, R.S. Rivlin, Arch. Rational Mech. Anal. 62, 237 (1976)

7. A. San Andres, A.Z. Szeri, J. Appl. Mech. 51(12), 869 (1984)

8. D.L. Young, C.L. Chiu, C.M. Fan, C.C. Tsai, Y.C. Lin, Eur. J. Mec. B-Fluids 25, 877 (2006)

9. P. Zhang, X. Zhang, Mathematical Problems in Engineering, Article ID 451546 (2014)

10. S. Meena, P. Kandaswamy, L. Debnath, Int. J. Math. Math. Sci. 25(2), 93 (2001)

11. L.M. de Socio, L. Marino, Eur. J. Mec. B-Fluids 22, 85 (2003)

12. G.L. Morini, M. Spiga, J. Heat Transfer 121(4), 1083 (1999)

13. M. Avci, O.A. Aydin, C.R. Mecanique 324, 164 (2006)

14. G.L. Morini, M. Spiga, J. Heat Transfer, 129(3), 308 (2007)

15. T.J. Teleszewski, S.A. Sorko, Procedia Engineering 157, 171 (2016)

16. T.J. Teleszewski, S.A. Sorko, Journal of Numerical Methods for Heat \& Fluid Flow, 28(1), 220 (2018) 
17. J.H.C. Araujo, V. Ruas, A.S. Vargas, Int. J. Numer. Methods Fluids 11, 849 (1990)

18. N. Ashrafi, A. Hazbavi, Arch. Appl. Mech. 83(12), 1739 (2013)

19. A. Hazbavi, ASME. J. Thermal Sci. Eng. Appl., 8(2), 021020-021020-9 2016 